\title{
Measurement of Glomerular Filtration Rate With Magnetic Resonance Imaging: Principles, Limitations, and Expectations
}

\author{
AQ: 1 Nicolas Grenier, ${ }^{, \dagger}$ losif Mendichovszky, ${ }^{\ddagger}$ Baudouin Denis de Seneville, ${ }^{*}$ Sébastien Roujol," \\ Pascal Desbarat, ${ }^{\S}$ Michael Pedersen," Kevin Wells," Jorgen Frokiaer,
}

\begin{abstract}
Glomerular filtration rate (GFR) is the most useful quantitative index of renal function and is used clinically as the gold standard of renal dysfunction. Follow-up of patients with impaired renal function requires reliable measurements of GFR. Thus, serial GFR values estimated from magnetic resonance imaging (MRI) would be worthwhile if easy to obtain, accurate, and reproducible. Nuclear medicine-based techniques remain at present the reference method for quantification of GFR, but MRI should be able to compete in the near future. Several methods are available for measurement of GFR using MRI and freely filtered Gd-chelates: (1) measurement of the clearance of the agent using blood samplings; (2) measurement of the clearance of the agent using signal intensity changes within abdominal organs; (3) measurement of the extraction fraction of the agent; and (4) monitoring of tracer intrarenal kinetics. A high heterogeneity of protocols (e.g., in acquisition mode, dose of contrast, postprocessing techniques) is noted in the literature, reflecting the number of technical challenges that will have to be solved before to reach a consensus, and the reported accuracy and reproducibility are insufficient for justifying their use in clinical practice now.
\end{abstract}

$G$ lomerular filtration rate (GFR) is the most useful quanTitative index of renal function and is used clinically as the gold standard of renal dysfunction. Follow-up of patients with impaired renal function requires reliable measurements of GFR. Thus, serial GFR values estimated from magnetic resonance imaging (MRI) would be worthwhile if easy to obtain, accurate, and reproducible.

*Laboratoire d'Imagerie Moléculaire et Fonctionnelle, UMR-CNRS 5231 Université Victor Segalen-Bordeaux 2, Bordeaux, France.

$\uparrow$ Service d'Imagerie Diagnostique et Interventionnelle de l'Adulte, Groupe Hospitalier Pellegrin, Bordeaux, France.

¥Radiology \& Physics Unit, UCL Institute of Child Health, London, United Kingdom.

§Laboratoire Bordelais de Recherche en Informatique (LaBRI) UMR-CNRS 5800 Université Bordeaux 1, Bordeaux, France.

IIMR Research Center, Institute of Clinical Medicine, University of Aarhus, Aarhus, Denmark.

\|CVSSP, School of Electronics and Physical Sciences, University of Surrey, Surrey, United Kingdom.

\#Department of Clinical Physiology and Nuclear Medicine, Aarhus University Hospital-Skejby, Aarhus, Denmark.

Address reprint requests to Nicolas Grenier, Service d'Imagerie Diagnostique et Interventionnelle de l'Adulte, Groupe Hospitalier Pellegrin, Place Amelie Raba Leon, 33076 Bordeaux, France. E-mail: nicolas.grenier@ chu-bordeaux.fr
The commonest method used clinically for accurate GFR measurement is assessment of the plasma disappearance of a substance that is excreted from the body exclusively by glomerular filtration with no tubular reabsorption nor tubular excretion, for example, ethylenediamine tetraacetic acid (EDTA) or iothalamate. Most of the time, these standard methods are underused because they are quite time consuming and require several blood/urinary samplings. Therefore, a quantitative method based on tracer intrarenal kinetics obtained rapidly without blood and/or urine samplings coupled with a morphological evaluation of the kidneys and the entire excretory system would be extremely useful in clinical management of patients with renal disease.

Low molecular weight gadolinium (Gd)-chelates, such as Gd-DOTA or Gd-DTPA, all have a predominant renal elimination (approximately 98\%) by glomerular filtration without any tubular secretion or reabsorption. Therefore, these agents can be considered as glomerular tracers just like inulin, EDTA, or iodine contrast agents and can conveniently be used to calculate GFR with MRI.

Such functional approaches using MRI have received attention for many years, ${ }^{1}$ but the clinical applications are nowadays limited to semiquantitative evaluation because of several reasons. First, it is difficult to obtain accurate and 
reproducible information from the entire kidneys because of respiratory movements and to magnetic susceptibility artifacts arising from the surrounding bowel; second, the complex relationship between the observed MR signal changes and the concentration of the contrast agent makes quantitative analysis difficult; third, the different kinetic models that are proposed to calculate GFR from MRI data have to be validated; and fourth, the reported accuracy of these techniques is still unsatisfactory. Therefore, nuclear medicinebased techniques remain at present the reference method for quantification of GFR but MRI should be able to compete in the near future.

Several methods are available for measurement of GFR using MRI and freely filtered Gd-chelates: (1) measurement of the clearance of the agent using blood samplings; (2) measurement of the clearance of the agent using signal intensity (SI) changes within abdominal organs; (3) measurement of the extraction fraction of the agent; and (4) monitoring of tracer intrarenal kinetics.

\section{Determination of Gd-Chelate Concentration}

Quantification of physiological parameters such as GFR using contrast-enhanced MRI requires the conversion of SI changes into concentrations changes. The relationship between SI measured on MR images and Gd-chelate concentration is not linear due to T2 and T2* effects occurring at high concentrations. However, there is a linear relationship between and the $\mathrm{T} 1$ relaxation rate $(\mathrm{R} 1=1 / \mathrm{T} 1)$ and $\mathrm{Gd}$-chelate concentration. From Rl changes, quantification of the concentration of a contrast agent is possible according to the following linear relationship:

$$
C=\left(\mathrm{R} 1-\mathrm{Rl}_{0}\right) / \mathrm{r}
$$

where $R l_{0}$ is the bulk $\mathrm{R} 1$ relaxivity in the medium or the tissue without contrast agent and $r$ is the specific relaxivity of the agent.

Dynamic Rl change values can be obtained either indirectly using conversion methods from SI, or directly by measuring R1 instead of SI with specific sequences. The indirect methods include the following:

- calibration using a phantom of several tubes, filled with Gd-solutions at various concentrations, and imaged with the same pulse-sequence as the one used for the in vivo study. The acquired SI values are plotted against measured Rl values, and a polynomial fit is made to obtain a calibration curve. A recent study showed that this approach provides results that correlate well with those obtained by a reference technique. ${ }^{2}$ However, this approach assumes that the relaxivity of the contrast agent is equivalent in solution and in tissues and that the difference between room and body temperature will have little effect.

- an analytical calibration based on the equation driven by the sequence used, ${ }^{3}$ which unfortunately is not straightforward. In addition, some sequences may not even have a an analytical formula that can be used for this method. ${ }^{4}$

- Rl of a tissue also can be measured directly using an appropriate pulse-sequence, such as a gradient-echo one with several flip angle values, ${ }^{5}$ or using a very fast inversion recovery pulse-sequence with several inversion times based on the Look-Locker method. ${ }^{6}$

\section{Measurement of Gd-Pasma Clearance}

Because there is a linear relationship between Gd-chelate concentration and the $\mathrm{Tl}$ relaxation rate $(\mathrm{R} 1=1 / \mathrm{T} 1)$, changes in $\mathrm{R} 1$ can be used for absolute quantification of the concentration of a contrast agent. Choyke and coworkers ${ }^{7}$ showed that measurement of Rl within serial blood samples after intravenous injection of Gd-DTPA was feasible, safe for the kidney, and reliable when compared with ${ }^{99} \mathrm{~m}$ Tc-DTPA clearance $(\mathrm{y}=1.04 \times 2.2$, $r=0.94$; Fig. 1). This method presents little advantage com- $\mathrm{Fl}$ pared with other methods because it is time-consuming and requires several blood samples.

\section{Measurement of Gd-Renal Clearance}

The renal clearance of a freely filtered Gd-chelate also can be calculated by measuring the slope clearance of the tracer from the extracellular fluid volume (ECFV) after an intravenous injection of the tracer. Using this principle, Boss and cowork$\mathrm{ers}^{8}$ measured, in 16 healthy volunteers, the SI changes, induced by the contrast agent $(0.05 \mathrm{mmol} / \mathrm{kg}$ of gadobutrol),

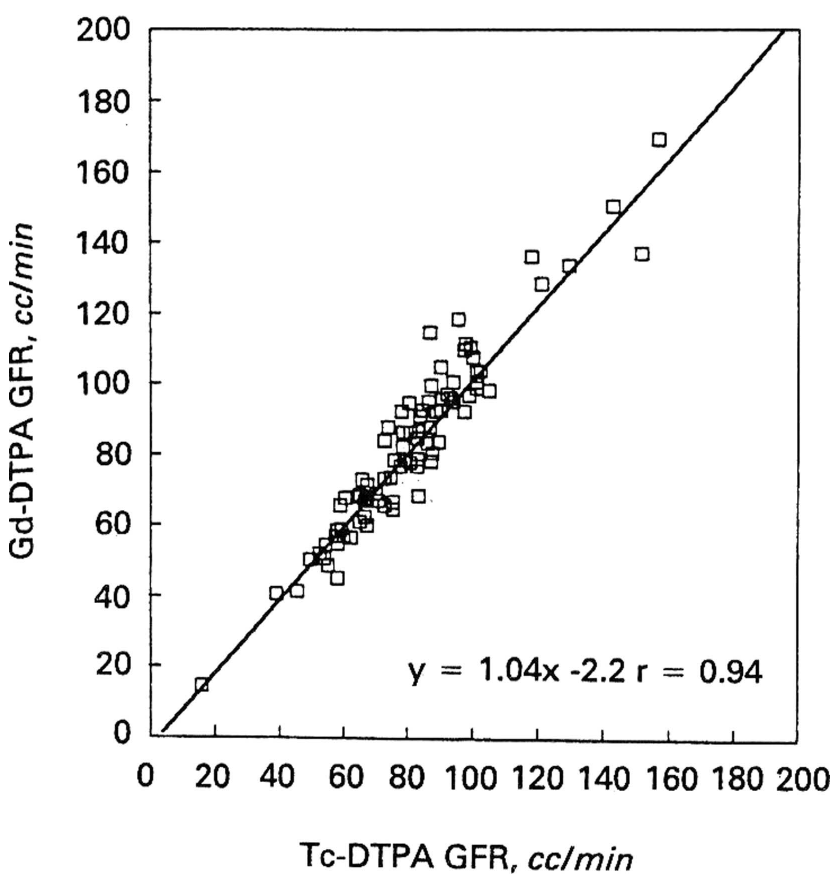

Figure 1 Measurement of plasma clearance using blood samplings. Comparison of Tc-DTPA derived GFR values to Gd-DTPA derived GFR values. (Reprinted with permission from Choyke et al. ${ }^{7}$ ) 
within several abdominal organs (liver, spleen, and renal cortex) during the course of 80 minutes. The results were compared with simultaneous iopromide clearance measurement as reference standard. The sequence used was a navigatorgated FLASH sequence $\left(\mathrm{TR}=498, \mathrm{TE}=1.25, \mathrm{FA}=8^{\circ}\right)$ with a nonselective magnetization preparation and a spoiler for heavy Tl-weighting. The GFR was calculated as the product of the ECFV (ECFV $=0.02154$. weight ${ }^{0.6469} \cdot$ height $^{0.3964}$ ) and the time constant of the second exponential phase $\left(\alpha_{2}\right)$. The best concordance between gadobutrol clearance and io-
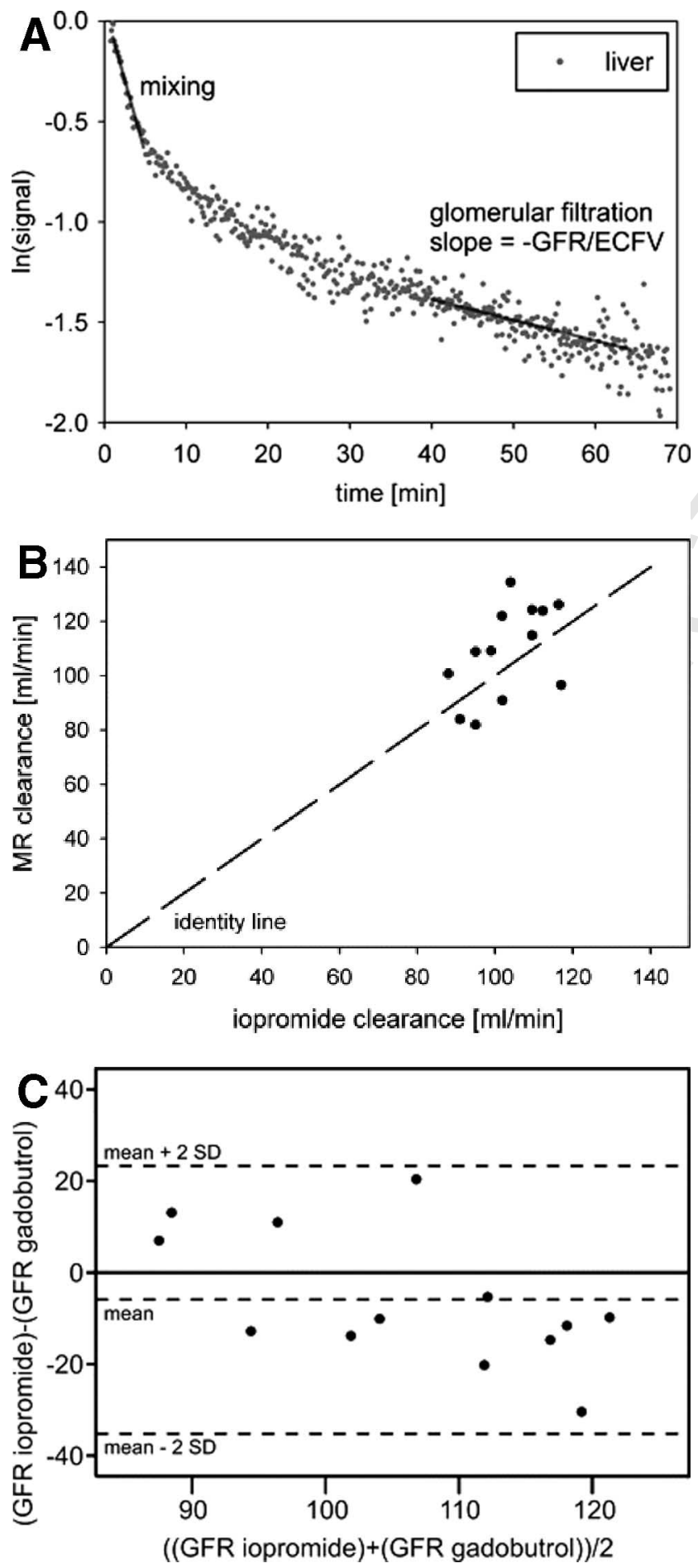

promide clearance was observed within the liver, the exponential fit being performed between 40 and 55 minutes after injection (with a mean paired difference of $-5.9 \mathrm{~mL} / \mathrm{min} /$ $1.73 \mathrm{~m}^{2} \pm 14.6$; Fig. 2). All measurement points were within \pm 2 standard deviation values but the maximum deviation form the reference GFR was 29\%. With such a wide deviation, this technique cannot be applied to the individual patient. The main drawbacks of this method are the length of MR acquisition (approximately 70 minutes), the sensitivity to body movements, and to the selected time intervals when the analysis is performed. More experience is required with this technique.

\section{Measurement of Extraction Fraction}

This method allows a quantification of a single kidney (SK) extraction fraction (EF), based on the measurement of $\mathrm{Tl}$ within flowing arterial and venous blood (Look and Locker method) during a continuous Gd infusion ${ }^{9-12}$ :

$$
\mathrm{EF}=\mathrm{Ca}-\mathrm{Cv} / \mathrm{Ca}
$$

where $\mathrm{Ca}$ and $\mathrm{Cv}$ are the arterial and venous Gd concentration respectively. Because of the linear relationship between relaxation rates and concentration of Gd, EF can be expressed alternatively as:

$$
\mathrm{EF}=((1 / \mathrm{Tla})-(1 / \mathrm{Tl} \mathrm{v})) /\left((1 / \mathrm{Tl} \mathrm{a})-\left(1 / \mathrm{Tl}_{0}\right)\right)
$$

where $\mathrm{Tl} \mathrm{a}$ is $\mathrm{Tl}$ in the renal artery, $\mathrm{Tl} \mathrm{v}$ in the vein and $\mathrm{T} 1_{0}$ in the blood without Gd. Once EF is calculated for each kidney, values of SKGFR can be calculated if RBF is known:

$$
\mathrm{SKGFR}=\mathrm{EF} \times \mathrm{RBF} \times(1-\mathrm{Hct})
$$

where RBF is the renal blood flow, usually measured with the cine-phase contrast method on the renal artery, and Hct is the level of hematocrit in the blood.

In practice, the $\mathrm{Tl}$ of the blood is measured in the infrarenal inferior vena cava for the arterial input (because the renal

Figure 2 Measurement of renal clearance. (A) Exponential decay on the logarithmically scaled time-signal intensity curve (displayed for liver parenchyma) indicates linear behavior. Two exponential phases can be distinguished owing to different slopes: the fast mixing phase immediately after contrast medium injection and the more prolonged phase, in which the signal intensity decrease is caused solely by glomerular filtration. The negative slope of this phase is equal to the GFR divided by the ECFV. (B and C) Normalized GFR measured by using gadobutrol clearance for MR evaluation of an ROI in the liver at 40-65 minutes after contrast medium injection is compared with iopromide clearance. (B) Graph shows a plot of the GFR values obtained by using both methods. (C) BlandAltman plot relates the difference between each measurement pair to the mean value. A mean difference in measurement pairs of $-5.9 \mathrm{~mL} / \mathrm{min} \pm 14.6$ was calculated. All measurement points are within \pm 2 standard deviations from the mean value. (Reprinted with permission from Boss et al. ${ }^{8}$ ) 
artery is too small) and on the renal vein for the venous 3 output (Fig. 3A). Preliminary animal studies have shown concordant results compared with those calculated from inulin clearance, but the correlation was 0.77 , which is inadequate for clinical use at this stage (Fig. 3B). ${ }^{13}$ No clinical application of this method has been published until now.
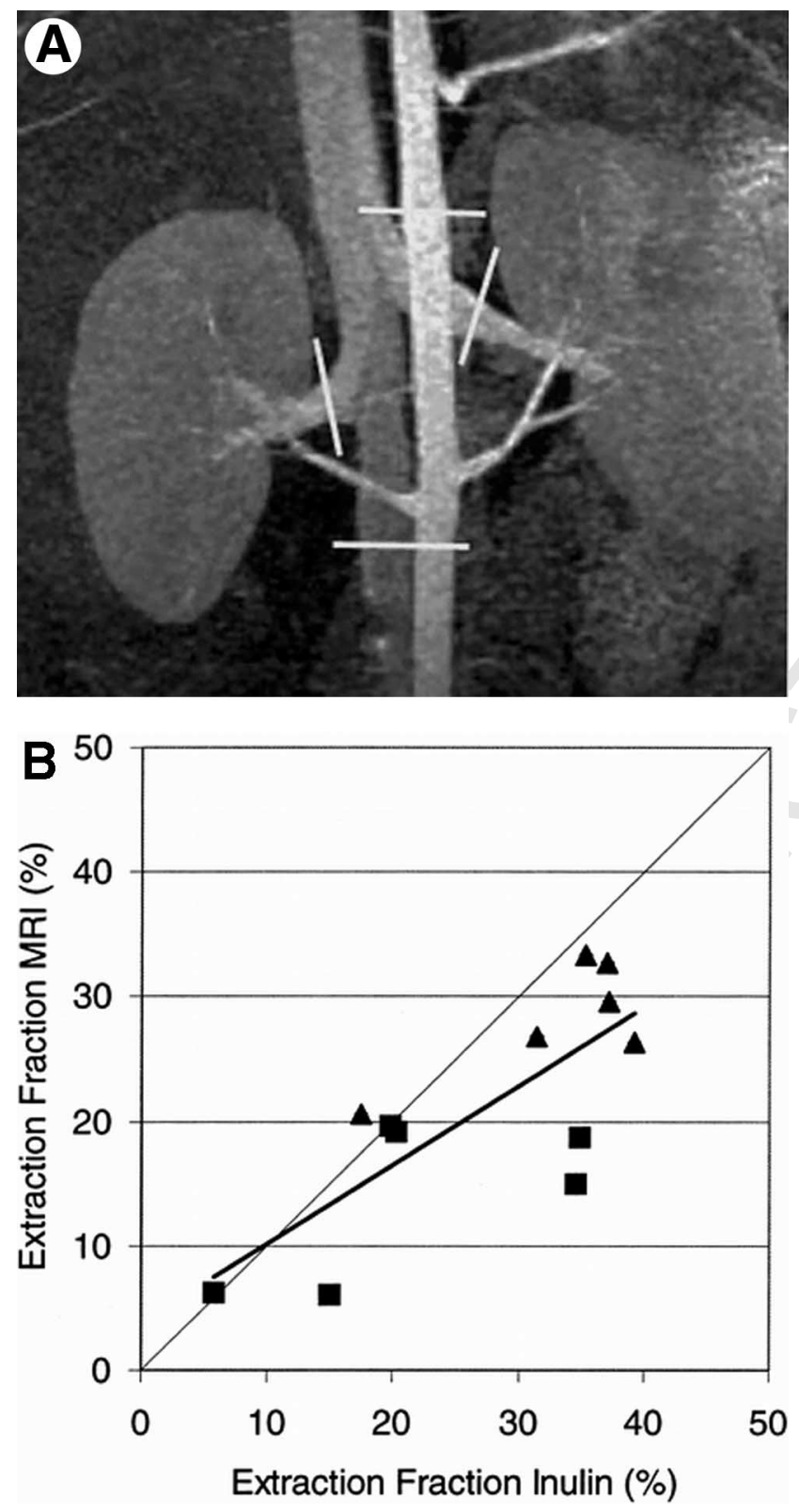

Figure 3 Measurement of extraction fraction. (A) Coronal maximum intensity projection image from contrast-enhanced 3-dimensional spoiled gradient-echo MR imaging (3.6/1.0; flip angle, 25). Lines indicate imaging planes for spiral $\mathrm{T} 1$ relaxation measurements. (B) Plot of single-kidney EF determined with inulin measurement and MR imaging. A statistically significant linear correlation between EF measured with MR imaging versus $\mathrm{EF}_{\mathrm{IN}}$ was found $\left(r^{2}=\right.$ 0.77). ( $\mathbf{\Delta})$ = value observed in kidney without RAS (right kidneys), $(\boldsymbol{\square})=$ value observed in kidney with RAS (left kidneys). (Reprinted with permission from Coulam et al. ${ }^{13}$ )

\section{Tracer Intrarenal Kinetics With Dynamic MRI}

This method is based on modeling of an MR renogram into 2 or more compartments obtained after intravenous injection of a bolus of Gd-chelate.

\section{Dynamic MR Acquisition}

A great heterogeneity for parameters of pulse-sequences, doses of injected Gd contrast, methods for conversion of signal intensity into concentration, postprocessing methods, and compartment models is still noted in the literature between the different groups and no consensus exists.

\section{Sequences}

All the pulse-sequences used must have a heavy T1-weighting and be fast enough to characterize the vascular phase of the tracer kinetic, which is necessary for assessment of the arterial input function. A reasonable Tl weighting can be accomplished using spoiled gradient-echo (type FLASH, i.e., fast low-angle shot) and RF-flip angles between $45^{\circ}$ and $90^{\circ}$, which provide a relative linear relationship between SI and Gd concentration. ${ }^{14}$ However, to obtain very short acquisition times and a heavy $\mathrm{Tl}$-weighting, a nonselective magnetization preparation has to be used, combined with very short TR and TE values and low flip angles. The preparation pulse is either realized as a saturation pulse $\left(90^{\circ}\right)$ or as an inversion pulse $\left(180^{\circ}\right)$, or alternatively, a more complex pulse scheme $\left(90^{\circ}-180^{\circ}\right)$ to get rid of the effects of arrhythmia on flow artifacts. ${ }^{15}$ Therefore, according to selected geometric parameters and the performance of the MRI system used, a compromise may be necessary.

\section{Dose of Contrast Agent}

The concentration of Gd within the kidney can be very high because ofwater reasbsorption in the proximal convoluted tubule and within the medulla. High concentrations also can be reached in the abdominal aorta during the bolus. Therefore, to avoid the $\mathrm{T} 2 *$ contribution of the agent occurring at high concentration, the injected dose must be lower than the regular clinical dose of $0.1 \mathrm{mmol} / \mathrm{kg}$, and the patient must be well hydrated. In the literature, the dose of gadolinium used varied from 2 to $16 \mathrm{~mL}$ (for adults), with the Gd being diluted to different volumes (in 2 studies, the Gd was diluted to a final injection volume of $60 \mathrm{~mL}$ ). The rate of injection in humans varied, being either automatic ( 1 or $2 \mathrm{~mL} / \mathrm{s}$ ) or manual. In the animal studies the dose was between 0.05 and $0.075 \mathrm{mmol} / \mathrm{kg}$ body weight with a manual injection technique. There is no consensus about the optimal dose to be used for that purpose. The choice between $0.025 \mathrm{mmol} / \mathrm{kg}$ and $0.05 \mathrm{mmol} / \mathrm{kg}$ depends on the level of signal-to-noise ratio obtained with the sequence and the system used.

\section{Acquisition Volume}

Some groups acquired only one median slice through both kidneys, and, calculating a mean GFR value, extrapolated this value to the entire renal volume to get a total GFR value. This approach is simpler, allowing a high temporal resolution, but neglects the probable heterogeneity of filtration 


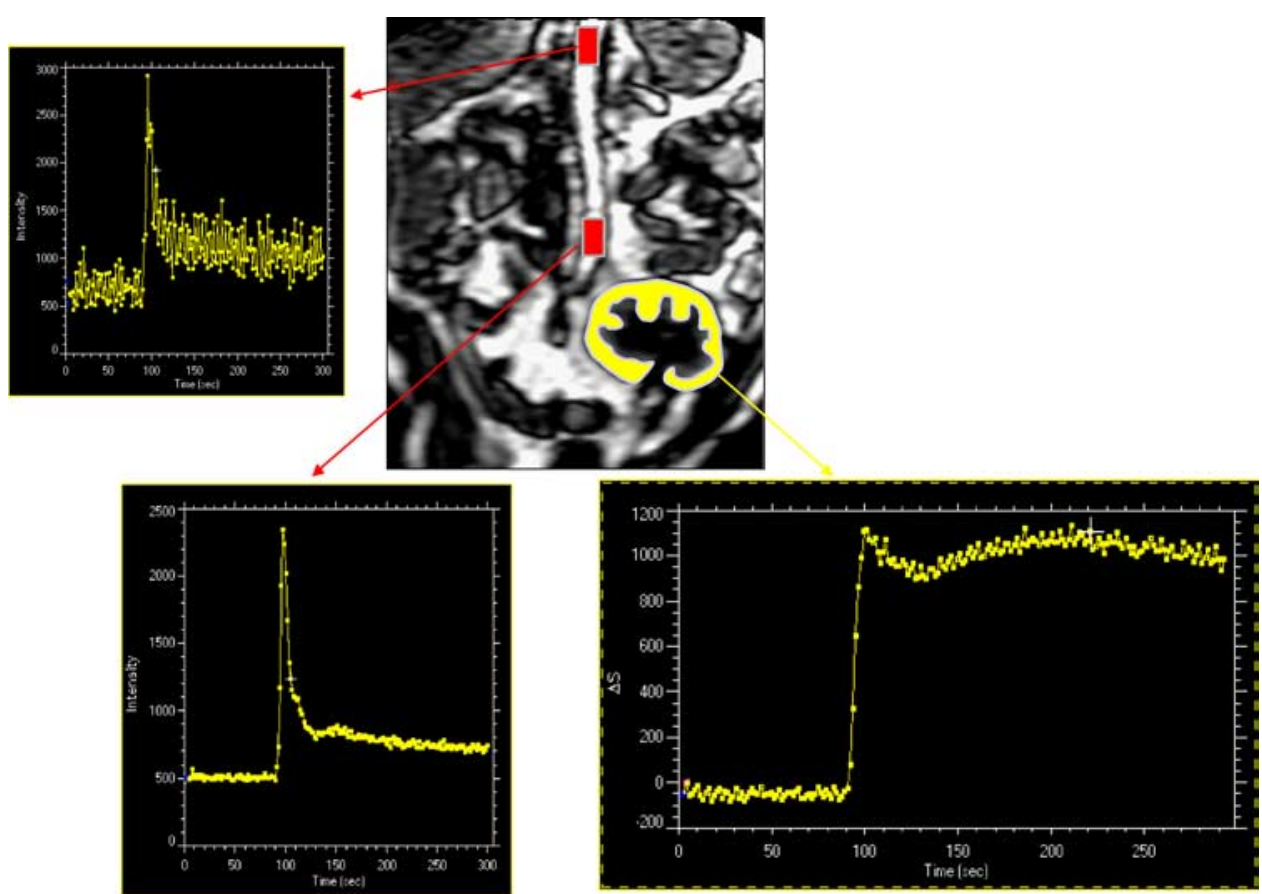

Figure 4 SI-time curves obtained on a renal transplant. The aortic curve, for characterization of the arterial input function, is altered by inflow effects only when sampling of SI is done at the top of the volume. The cortical SI time curve can be characterized by 3 phases: a first abrupt ascending segment followed by a first peak, corresponding to the "vascular-to-glomerular first-pass" or cortical vascular phase; a second slowly ascending segment, ended by a second peak, corresponding to the glomerulo-tubular phase; and a slowly descending segment, corresponding to the predominant excretory function and so-called "excretory phase."

function within the cortex. Also when dealing with diseased kidneys the assumption that the single slice accurately represents the entire kidney may be untrue. Therefore, others prefer to cover the entire parenchyma of each kidney, using multislice or 3D acquisitions, to be able to sum the GFR values of each slice, or even of each voxel in each slice. Comparison of these 2 approaches has never been performed.

\section{Acquisition Plane}

In the literature, the dynamic renal images are acquired either in an oblique-coronal plane passing through the long axis of the kidneys or in the transverse plane. Drawbacks of the later are the higher number of slices required for covering the entire kidneys, with a negative impact on the time resolution, the impossibility for movement correction, and the risk of severe inflow effects within the aorta altering the accuracy of the arterial input function.

\section{Arterial Input Function (AIF)}

Measurement of renal filtration requires an accurate sampling of the vascular phase of the enhancement to measure the AIF. The AIF is characterized by the SI changes within the suprarenal abdominal aorta. It is used for the different kinetic models to compensate for the noninstantaneous bolus injected into the blood. Not taking this into account will produce an overestimation of GFR because of recirculation of the agent within the vascular space. Sampling the AIF requires a high temporal resolution. Recent advancements in MR instrumentation and hardware facilitate coverage of the entire kidneys with several slices using 3D pulse-sequences while maintaining a temporal resolution around 1.5 seconds. ${ }^{16}$

Coronal acquisition, coupled with a nonselective saturation or inversion pulse, has the advantage to get rid of inflow effects if sampling of the aorta is far enough from the top of the imaging volume (Fig. 4). These effects are more difficult $\mathrm{F}$ to control in axial acquisitions.

\section{Postprocessing of Data}

If the time resolution of the pulse-sequence is sufficiently high, normal SI-time curve after Gd injection can be characterized by 3 phases (Fig. 4): a first abrupt ascending segment followed by a first peak, corresponding to the "vascular-toglomerular first-pass" or cortical vascular phase; a second slowly ascending segment, ended by a second peak, corresponding to the glomerulo-tubular phase; and a slowly descending segment, corresponding to the predominant excretory function and so-called "excretory phase." If the region of interest (ROI) is restricted to the cortex, the slope of the second ascending segment is reduced. If the ROI is restricted to the medulla, the curve is characterized by a biphasic ascending slope, corresponding to the vascular and the uptake phases, the later segment reflecting the tubular transit and progressive concentration, and is followed by the excretory phase.

These renal curves constitute the basis for functional measurements. Therefore, they have to be optimized to remove 
the artifacts introduced by respiratory movements during data acquisition, partial volume effects, separation of segments with different functional status and application of appropriate pharmacokinetic models. These tasks will require the development of specific postprocessing techniques.

\section{Movement Correction}

Because dynamic MR imaging of the kidneys is performed during free breathing, the main problem with postprocessing these images is correction of respiratory movements. Because gated sequences ${ }^{17}$ would lead to severe penalties in temporal resolution, most of the groups studying renal function either repositioned images manually, on a visual basis before sampling, or applied no correction at all. Absence of correction produces artifacts in time-intensity evolution which can lead to incorrect quantification.

Although problems related to movement can in principle be addressed with image registration algorithms ${ }^{18}$ applied in a postprocessing step, additional challenges arise from the fact that image amplitude and contrast change with time due to the transit of the contrast agent during bolus passage. A movement correction method developed in our group ${ }^{19}$ is derived from a method proposed by Sun and coworkers, ${ }^{20}$ allowing the estimation of a rigid transformation and correction for movement in renal MR data with subpixel accuracy. The principle is the following: although the relative intensities between tissues vary with time, edge orientation along tissue boundary is always parallel across the image sequence. A ROI restricted to the renal cortex is manually drawn by the radiologist on an early enhanced image (during the vascular phase of renal enhancement which enhances strongly and specifically the cortex). A binary mask (noted $m$ ) was constructed: pixels of the mask inside the cortex had a value of one, and outside a value of zero. The reference image set for motion estimation of all images was the image on which the ROI had been manually set. The displacement of the kidney $T$ was estimated by a 3 parameters rigid body model. Let $\theta_{\text {ref }}$ $(x, y)$ and $M_{r e f}(x, y)$ be respectively the edge orientation and the edge magnitude for the pixel $(x, y)$ obtained using a Sobel edge detector on the reference image. Identically, let $\theta_{\text {cur }}(x, y)$ and $M_{c u r}(x, y)$ be respectively the edge orientation and the edge magnitude for the pixel $(x, y)$ obtained on the image to register. An exhaustive search is performed to determine the parameters of the spatial transformation $T$ that maximize the following edge-based consistency metric:

$$
\begin{aligned}
& \gamma\left(I_{\text {ref }}(x, y), I_{\text {cur }}(T(x, y))\right) \\
& =\frac{\sum_{x, y \in m} M_{r e f}(x, y) \cdot M_{\text {cur }}(T(x, y))}{\sum_{x, y \in m} M_{r e f}(x, y) \cdot M_{\text {cur }}(T(x, y))}
\end{aligned}
$$

A multistep scheme is used to perform a subpixel registration while reducing enumeration of possible solutions. With help of this accurate estimate, the ROI could be propagated to all images of the time series.

As kidney displacement is assumed to be rigid, the estimated 2D motion is used to register automatically all other slices. If the motion is correctly determined, the kidney border would coincide with the contour of the original region of interest manually drawn by the radiologist around the kidney. An experienced operator could also review the re-aligned data set and manually correct these if necessary.

This algorithm could estimate successfully small motion and more than $20 \%$ of reduction on GFR uncertainty on transplanted kidneys Fig 5 and up to $60 \%$ on native kidneys, using Rutland-Patlak method.
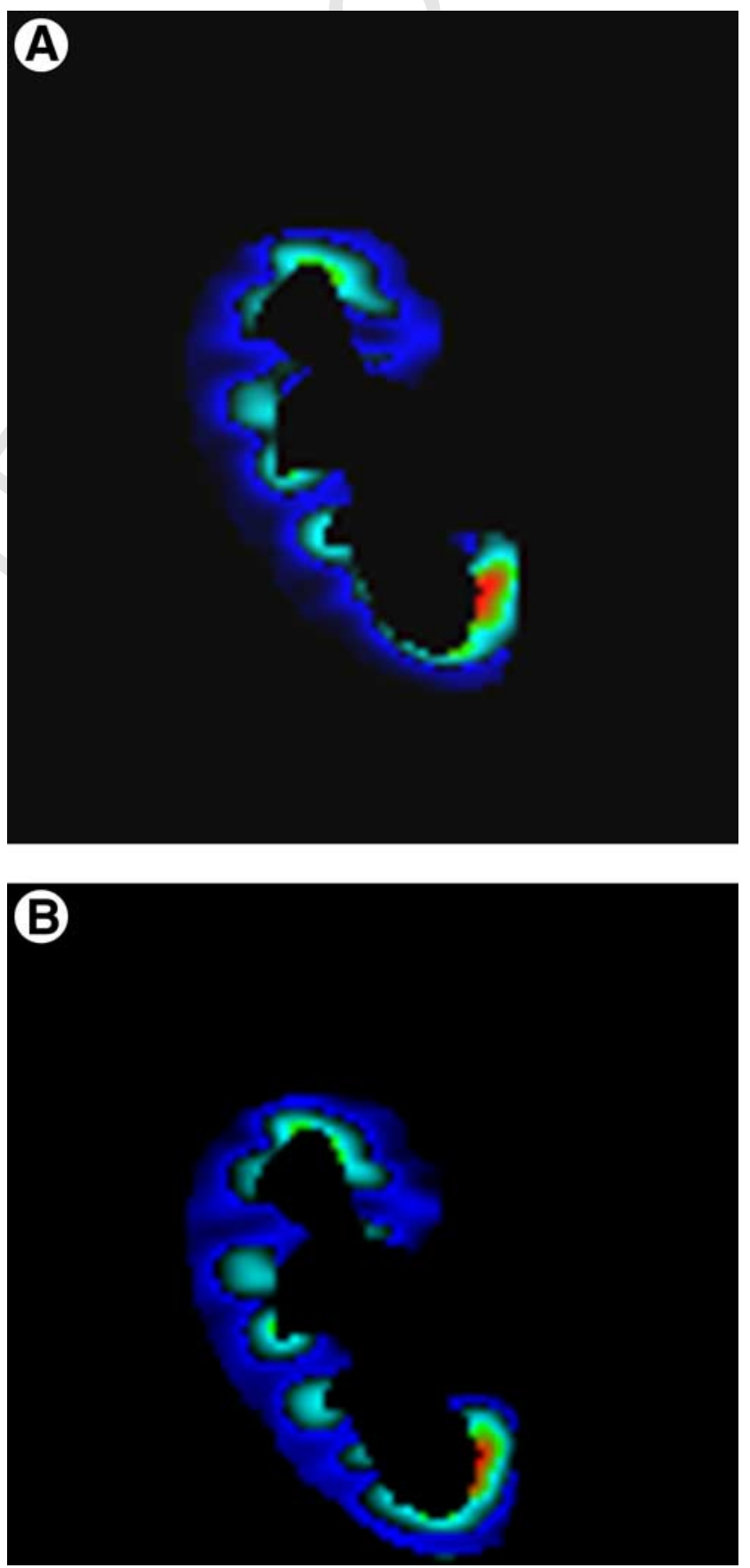

Figure 5 Color map of GFR, calculated on a voxel-by-voxel basis, using the Rutland-Patlak plot technique, without (A) and with (B) automatic motion correction. 
342AQ: 2 In the future,

343

344

345

346

347

348

349

350

351

352

353

354

355

356

357

358

359

360

361

362

363

364

365

366

367

368

369

370

371

372

373

374

375

376

377

378

379

380

381

382

383

384

385

386

387

388

389

390

391

392

393

394

395

396

397

398

399 volume artifacts. model.

\section{Renal and Cortex Segmentation}

In the clinical studies published in the literature, segmentation and ROI generation were generally done manually and the difficulties in this area were poorly addressed. Applying an adequate model to the renographic data requires an effective segmentation of renal compartments, cortex and medulla, to separate their respective function. Such a manual segmentation is time-consuming and therefore requires automatic methods. Furthermore, to avoid interoperator variability, several segmentations by hand must be done for each renographic data set. A recent review has developed and discussed extensively the principles and the difficulties on segmentation and on renal ROI generation. ${ }^{21}$ Several groups of methods are offered: simple thresholding, boundary-based techniques, region-based techniques, and other types of semiautomated methods.

These algorithms are only geometry-based and, therefore, their efficiency is correlated with image contrast and SNR. A first approach to develop a high-level MR renography segmentation algorithm using graphs as been introduced. ${ }^{22}$ If the result of the segmentation of the cortex and the medulla is encouraging, it could be further improved by taking into account the topology of the kidney. To do this, the graph representation of the kidney has to be oriented, resulting in a combinatorial map. ${ }^{23}$ This approach should allow an accurate segmentation by considering the cortex as a connected set of voxels and medulla as "holes" (lack of signal). In the future, a real 3D segmentation has to be performed. Computation of a complete 3D renal reconstruction will be necessary to follow the cortex boundary in $3 \mathrm{D}$ and to avoid partial

\section{Partial Volume Effects}

The partial volume effect occurs where signals from 2 or more tissues combine to produce a single image intensity value within a particular voxel. This effect becomes evident mostly at the boundaries between tissues, and is present to a greater or lesser degree in all imaging modalities. In functional MRI acquisitions, where spatial resolution is killed for better temporal resolution (such as magnetic resonance renography) the partial volume effect is likely to be significant. This potentially impacts on ROI selection, and its interpretation for subsequent GFR estimation. Work recently presented suggested that voxels of the right kidney cortex adjacent to the liver may have as much as $20 \%$ contribution from the liver. (Rodriguez Gutierrez D, Diaz Montesdeoca O, Wells K, et al: Partial volume correction for magnetic resonance renography studies. British Chapter of the ISMRM, 12th Annual Meeting, Guilford, August 23-25, 2006).

\section{Compartment Models}

The ideal model for the analysis of the renal dynamic contrast-enhanced MRI data in the kidney remains to be worked out. Most of the reviewed MRI methods used a 2-compartment model, although the more recent publication by Lee and coworkers ${ }^{24}$ proposed a more complex compartment
The Rutland-Patlak plot technique is a graphical method based on a 2-compartmental model with the assumptions that the rate of change of concentration in the kidney during the clearance phase is constant if the amount of contrast agent is taken into account during this period. ${ }^{25}$ This method has been applied to MRI by several groups. ${ }^{26-30}$ Generally, the interstitial space is neglected, and Gd is considered to mix freely between the capillary and the glomerular compartments. This assumption may, however, fail in situations where the interstitial space is expanded (pyelonephritis and some examples of acute renal failure) which may lead to overestimation of SKGFR. The assumption that no contrast agent leaves the ROI during the sampling period may justify the use of ROIs encompassing both cortex and medulla. The model is realized as an $x-y$ plot (the Rutland-Patlak plot) using the ratio of the renal concentration/aortic concentration plotted against the ratio of the integral of aortic concentration/aortic concentration. When applied to the second phase of the signal intensity-time curve (glomerulo-tubular uptake), this plot leads to a straight line, with a slope proportional to the renal clearance, and an intercept with the y-axis proportional to the cortical blood volume.

The cortical compartment model ${ }^{26,27}$ is another 2-compartment model confined to the cortex. It derives from the Rutland-Patlak model but differs by the constraints that the outflow from the tubules, during the sampling period, is taken into account. This makes it possible to draw ROIs strictly limited to the cortex. The aortic curve is shifted and dispersed taking into account the transit time of the contrast agent between the aorta and the kidney and the tracer dispersion in the glomeruli. ${ }^{26}$ Calculation of the residue function, (ie, the deconvolution of $\mathrm{C}(\mathrm{t})$ with $\mathrm{AIF}(\mathrm{t})$ ) then exhibits 3 sequential peaks (successively glomerular, proximal tubule, distal tubule) and 2 rate constants, kin and kout that describe the flow into and out of the proximal tubule, meaning that kin represents SKGFR. SKGFR can be calculated according to the following equation:

SKGFR = maximal slope of proximal tubule peak/

$$
\text { C(vasc) max }
$$

To calculate $\mathrm{C}$ (vasc)max, the maximum of the vascular peak was divided by the RBV (see chapter on perfusion) to take into account that the contrast agent remains in the extravascular space. This method has provided more accurate results than Rutland-Patlak model in an experimental study in rabbits, using 51Cr-EDTA as a gold standard. ${ }^{26}$

The multicompartmental mode ${ }^{24}$ includes 3 cortical compartments (glomerular, capillary, and proximal convoluted tubules, as in previous model), 3 medullary compartments (loops of Henle, distal convoluted tubules, collecting ducts), and the collecting system. Despite being complex, this model has the advantage of assessing some important tubular physiological parameters. Applied to the passage of Gd into the first 2 compartments, the model allows calculation of GFR.

\section{Calculation of Single Kidney GFR}

The total SKGFR (in $\mathrm{mL} / \mathrm{min}$ ) can be obtained either by multiplying the mean GFR value, measured on a cortical region of 


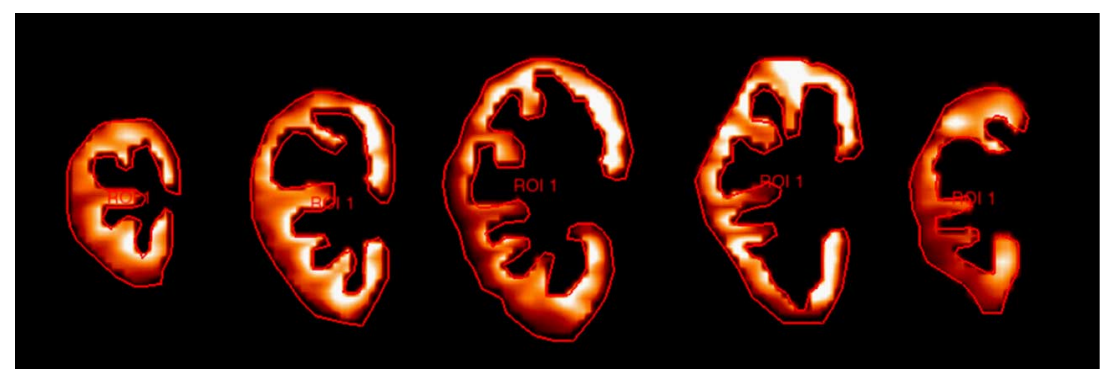

400

401

402

403

404

405

406

407

408

Figure 6 Color maps of GFR, calculated on a voxel-by-voxel basis, using the Rutland-Patlak plot technique, on the 5 slices of a renal transplant. The cortex was segmented manually.

interest, by the functional renal volume being either the renal cortex ${ }^{27}$ or the total renal volume. ${ }^{5}$ In the normal kidney, the cortical volume can also be considered as $70 \%$ of the total kidney volume. ${ }^{31}$

Another method for single kidney GFR calculation consists in modeling, on voxel-by-voxel basis, each MR-slice of the kidney (providing renal functional maps) and to add the 66 values from each voxel of each slice (Fig. 6). This method has the advantage it takes into account the heterogeneity of filtration within the organ. To our knowledge, these 2 approaches have still to be compared.

\section{Accuracy and Reproducibility}

After multiple blood sampling (2-4 hours) after a single intravenous bolus injection of ${ }^{51} \mathrm{Cr}$-EDTA and a single compartment analysis for the measurements of GFR, Chantler and coworkers ${ }^{32}$ showed a coefficient of variation $(\mathrm{CV})$ of 3.9\%. Brøchner-Mortensen and Rødbro ${ }^{33}$ showed a precision of $5.5 \%$ and a reproducibility $(\mathrm{CV})$ of $4.1 \%$ in patients with a plasma clearance $>30 \mathrm{~mL} / \mathrm{min}$, whereas with a plasma clearance $<30 \mathrm{~mL} / \mathrm{min}$, a precision of $11.6 \%$, and a reproducibility of $11.5 \%$ were obtained. Blake and coworkers ${ }^{34}$ showed that the long-term precision of GFR based on the ${ }^{51} \mathrm{Cr}$-EDTA clearance had a CV of $9.8 \%$.

Studies using dynamic contrast-enhanced MRI should be accurate and reproducible when compared with a gold standard technique. Several published papers conclude that Gdenhanced MRI provides a reliable estimate of GFR and is suitable for use in both clinical and experimental settings. ${ }^{29,30,35,36}$ However, a recent analysis of the literature showed a great heterogeneity of protocols (e.g., in acquisition mode, dose of contrast, postprocessing techniques) and that these studies presented regression coefficient values that are generally considered inadequate for replacing one technique with an accepted reference method in biomedical sciences. ${ }^{37}$ The claims in the current literature that using MRI "Gd DTPA clearance provides a good estimate of glomerular filtration ${ }^{35}$ " are not supported and the conclusion that no "approach used provides a wholly satisfactory measure of renal function" ${ }^{5}$ " is more accurate. The single publication on reproducibility is not encouraging because the absolute values have a wide $\%$ reproducibility, which is not clinically useful. ${ }^{38}$

One possible explanation for the "poor" published results on both reproducibility and accuracy is that there is a biological variation in GFR but, obviously, it is unacceptable to introduce methods that have a lower accuracy and reproducibility than existing methods. Only one study undertook the 99mTc-DTPA clearance during the Gd-MRI ${ }^{24}$ but even these results with a complex mathematical model-based SKGFR the $95 \%$ confidence interval varied between 5.8 and 17.9 $\mathrm{mL} / \mathrm{min}$ compared with the reference technique.

In summary, although MRI has a great potential to provide accurate and reproducible results for measurement of glomerular filtration, none of the proposed methods has been sufficiently validated to be recommended as a routine clinical/ research tool. The high heterogeneity of protocols reflects the number of technical challenges that will have to be solved before to reach a consensus.

\section{References}

1. Bennett HF, Li D: MR imaging of renal function. Magn Reson Imaging Clin N Am 5:107-126, 1997

2. Bokacheva L, Rusinek H, Chen Q, et al: Quantitative determination of Gd-DTPA concentration in Tl-weighted MR renography studies. Magn Reson Med 57:1012-1018, 2007

3. Pedersen M, Shi Y, Anderson P, et al: Quantitation of differential renal blood flow and renal function using dynamic contrast-enhanced MRI in rats. Magn Reson Med 51:510-517, 2004

4. Rusinek H, Lee VS, Johnson G: Optimal dose of Gd-DTPA in dynamic MR studies. Magn Reson Med 46:312-316, 2001

5. Buckley DL, Shurrab AE, Cheung CM, et al: Measurement of single kidney function using dynamic contrast-enhanced MRI: comparison of two models in human subjects. J Magn Reson Imaging 24:1117-1123, 2006

6. ZhengJ, Venkatesan R, Haacke EM, et al: Accuracy of Tl measurements at high temporal resolution: feasibility of dynamic measurement of blood $\mathrm{Tl}$ after contrast administration. J Magn Reson Imaging 10:576581, 1999

7. Choyke PL, Austin HA, Frank JA, et al: Hydrated clearance of gadolinium-DTPA as a measurement of glomerular filtration rate. Kidney Int 41:1595-1598, 1992

8. Boss A, Martirosian P, Gehrmann M, et al: Quantitative assessment of glomerular filtration rate with MR gadolinium slope clearance measurements: a phase I trial. Radiology 242:783-790, 2007

9. Dumoulin CL, Buonocore MH, Opsahl LR, et al: Noninvasive measurement of renal hemodynamic functions using gadolinium enhanced magnetic resonance imaging. Magn Reson Med 32:370-378, 1994

10. Niendorf ER, Santyr GE, Brazy PC, et al: Measurement of Gd-DTPA dialysis clearance rates by using a look-locker imaging technique. Magn Reson Med 36:571-578, 1996

11. Niendorf ER, Grist TM, Frayne R, et al: Rapid measurement of GdDTPA extraction fraction in a dialysis system using echo-planar imaging. Med Phys 24:1907-1913, 1997 
12. Niendorf ER, Grist TM, Lee FT Jr, et al: Rapid in vivo measurement of single-kidney extraction fraction and glomerular filtration rate with MR imaging. Radiology 206:791-798, 1998

13. Coulam $\mathrm{CH}$, Lee JH, Wedding KL, et al: Noninvasive measurement of extraction fraction and single-kidney glomerular filtration rate with MR imaging in swine with surgically created renal arterial stenoses. Radiology 223:76-82, 2002

14. Rohrschneider WK, Haufe S, Wiesel M, et al: Functional and morphologic evaluation of congenital urinary tract dilatation by using combined static-dynamic MR urography: Findings in kidneys with a single collecting system. Radiology 224:683-694, 2002

15. Montet X, Ivancevic MK, Belenger J, et al: Noninvasive measurement of absolute renal perfusion by contrast medium-enhanced magnetic resonance imaging. Invest Radiol 38:584-592, 2003

16. Pedersen M, Frokier J, Grenier N: Quantitative measurement of renal function using contrast enhanced MRI: an initial experience. Proceedings of Int Scientific Meeting Radionucl Nephr-Urol (ISCORN), La Baule, 2004. (Poster M15)

17. Moricawa S, Inubushi T, Kurumi Y, et al: Feasibility of simple respiratory triggering in MR-guided interventional procedures for liver tumors under general anesthesia. Proceedings of ISMRM 10th Annual Meeting, Hawaii, 2002

18. Maintz JBA, Viergever MA: A survey of medical image registration. Med Image Analysis 2:1-36, 1998

19. Denis de Senneville B, Mendichovszky I, Roujol S, et al: Improvement of MRI- functional measurement with semi-automatic movement correction in native and transplanted kidneys. Magn Reson Med

20. Sun Y, Jolly MP, Moura JMF: Integrated registration of dynamic renal perfusion MR images. Proceedings of ICIP-IEEE. Singapore, 2004

21. Michoux N, Vallee JP, Pechere-Bertschi A, et al: Analysis of contrastenhanced MR images to assess renal function. Magma 19:167-179, 2006

22. Rusinek H, Boykov Y, Kaur M, et al: Performance of an automated segmentation algorithm for 3D MR renography. Magn Reson Med 57: 1159-1167, 2007

23. Braquelaire JP, Brun L: Image segmentation with topological maps and interpixel representation. J Visual Communication and Image Representation 9:62-79, 1998

24. Lee VS, Rusinek H, Bokacheva L, et al: Renal function measurements from MR renography and a simplified multicompartmental model. Am J Physiol 292:F1548-F1559, 2007
25. Patlak CS, Blasberg RG, Fenstermacher JD: Graphical evaluation of blood-to-brain transfer constants from multiple-time uptake data. J Cereb Blood Flow Metab 3:1-7, 1983

26. Annet L, Hermoye L, Peeters F, et al: Glomerular filtration rate: assessment with dynamic contrast-enhanced MRI and a cortical-compartment model in the rabbit kidney. J Magn Reson Imaging 20:843-849, 2004

27. Hermoye L, Annet L, Lemmerling P, et al: Calculation of the renal perfusion and glomerular filtration rate from the renal impulse response obtained with MRI. Magn Reson Med 51:1017-1025, 2004

28. Pedersen M, Dissing T, Deding D, et al: MR renography based on contrast-enhanced Tl-mapping. Proceedings of Medicine ISfMRi. International Society for Magnetic Resonance in Medicine, Miami, FL, May 7-13 2005, p 526

29. Hackstein N, Cengiz H, Rau WS: Contrast media clearance in a single kidney measured on multiphasic helical CT: Results in 50 patients without acute renal disorder. AJR Am J Roentgenol 178:111-118, 2002

30. Hackstein N, Heckrodt J, Rau WS: Measurement of single-kidney glomerular filtration rate using a contrast-enhanced dynamic gradientecho sequence and the Rutland-Patlak plot technique. J Magn Reson Imaging 18:714-725, 2003

31. Hegedus V, Faarup P: Cortical volume of the normal human kidney. Correlated angiographic and morphologic investigations. Acta Radiol Diagn (Stockh) 12:481-496, 1972

32. Chantler C, Barratt TM: Estimation of glomerular filtration rate from plasma clearance of 51-chromium edetic acid. Arch Dis Child 47:613617, 1972

33. Brochner-Mortensen J, Rodbro P: Selection of routine method for determination of glomerular filtration rate in adult patients. Scand J Clin Lab Invest 36:35-43, 1976

34. Blake GM, Roe D, Lazarus CR: Long-term precision of glomerular filtration rate measurements using 51Cr-EDTA plasma clearance. Nucl Med Commun 18:776-784, 1997

35. Laurent D, Poirier K, Wasvary J, et al: Effect of essential hypertension on kidney function as measured in rat by dynamic MRI. Magn Reson Med 47:127-134, 2002

36. Lee VS, Rusinek H, Noz ME, et al: Dynamic three-dimensional MR renography for the measurement of single kidney function: Initial experience. Radiology 227:289-294, 2003

37. Mendichovszky I, Pedersen M, Frøkiær J, et al: How accurate is dynamic contrast-enhanced MRI in the assessment of renal glomerular filtration rate? A critical appraisal. J Magn Reson Imaging

38. Jorgensen B, Keller AK, Radvanska E, et al: Reproducibility of contrast enhanced magnetic resonance renography in adolescents. J Urol 176: 1171-1176, 2006 